\title{
Criação de um Aplicativo de Realidade Aumentada para Dispositivos Móveis Destinado ao Uso em Práticas Educativas
}

\author{
Cibelle Amorim Martins ${ }^{1}$, Jonas Rocha Araújo ${ }^{2}$, Rodolfo Araújo de Carvalho² \\ ${ }^{1}$ Centro de Educação - Universidade Federal do Rio Grande do Norte (UFRN) \\ ${ }^{2}$ Instituto Metrópole Digital - Universidade Federal do Rio Grande do Norte (UFRN) \\ Natal - RN - Brasil \\ \{rodolfooac, jonasra4,\}@gmail.com \\ cibelle.amorim@ce.ufrn.br
}

\begin{abstract}
The emergence of new technologies makes it possible to increase the interactivity of educational elements with users, creating innumerable ways to stimulate learning. This has made the learning process more participatory and motivating. The objective of this paper is to describe the development of the RALTE application that occurred in the Laboratory of Educational Technology (LTE) of the Education Center (CE) of the Federal University of Rio Grande do Norte (UFRN), which uses the Reality augmented, One of the possibilities of innovation of educational practices.
\end{abstract}

Resumo. O surgimento de novas tecnologias torna possivel o aumento da interatividade de elementos educacionais com os usuários, criando inúmeras formas de estimular o aprendizado. Isso tem tornado o processo de aprendizagem mais participativo e motivador. $O$ objetivo deste trabalho é relatar o desenvolvimento do aplicativo RALTE que ocorreu no Laboratório de Tecnologia Educacional (LTE), do Centro de Educação (CE) da Universidade Federal do Rio Grande do Norte (UFRN), que utiliza da tecnologia de realidade aumentada, como uma das possibilidades de inovação das práticas educativas.

\section{Introdução}

Discussões relacionadas ao uso de recursos computacionais no processo de ensino e aprendizagem se tornaram frequentes nos últimos anos. O surgimento de novas tecnologias têm vislumbrado avanços nas formas tradicionais de ensino, possibilitando um aprendizado mais rico e envolvente por parte dos alunos, com o uso de mecanismos lúdicos, interativos e dinâmicos, alterando a forma como o professor proporciona a construção do conhecimento pelo discente.

Já é consenso entre profissionais e pesquisadores da educação que os recursos educacionais digitais podem facilitar o processo de ensino e aprendizagem de diversas maneiras. Nas aulas teóricas, por exemplo, podem auxiliar o professor na construção de conceitos que seriam de difícil explicação apenas por meio do uso de apresentações orais. Realidade Aumentada (RA) é uma tecnologia [Silva, Oliveira, \& Giraldi 2003] que vem sendo amplamente investigada para o seu uso educacional. Isso ocorre devido 
VI Congresso Brasileiro de Informática na Educação (CBIE 2017)

Anais dos Workshops do VI Congresso Brasileiro de Informática na Educação (WCBIE 2017)

ao potencial que essa tecnologia tem de auxiliar na construção do conhecimento, podendo ser utilizada nas mais diversas áreas do ensino [Martins \& Guimarães 2012].

A utilização da RA necessita ainda passar por processos de desenvolvimento técnico das ferramentas de marcação, como webcam ou smartphone, para fins educacionais. Além disso, para que essa tecnologia atenda à fins educativos, demanda-se objetivos claros e parâmetros didático-pedagógicos para os objetos de aprendizagem, a fim de enriquecer os recursos utilizados na construção do conhecimento [Yuen, YaoYuneyong e Johnson 2013], justificando o seu uso nessas práticas em sala de aula. $\mathrm{O}$ uso de RA para a produção do conhecimento apresenta importância devido às suas características básicas de interação, movimentação e concentração, simultaneamente com o estímulo motivacional provocado pela experiência que esse tipo de tecnologia proporciona.

Buscando contribuir com o desenvolvimento e integração desta tecnologia no contexto educacional, o Laboratório de Tecnologia Educacional (LTE), do Centro de Educação (CE) da Universidade Federal do Rio Grande do Norte (UFRN), iniciou um processo de estudos e pesquisas das ferramentas para o desenvolvimento de um aplicativo de RA, denominado RALTE. Nesse sentido, busca mostrar que a RA é uma tecnologia acessível, de baixo custo, sendo um ponto relevante para a sua disseminação.

\section{Realidade Aumentada}

A RA é definida pela apresentação de objetos virtuais trazidas à cena do ambiente do mundo físico e real, como forma de auxiliar na visualização, sendo que esta abordagem é realizada por meio de um dispositivo tecnológico que torna a visão do usuário "aumentada" [Azuma 1997]. Na Figura 1, é apresentado um exemplo de RA por intermédio de um smartphone.

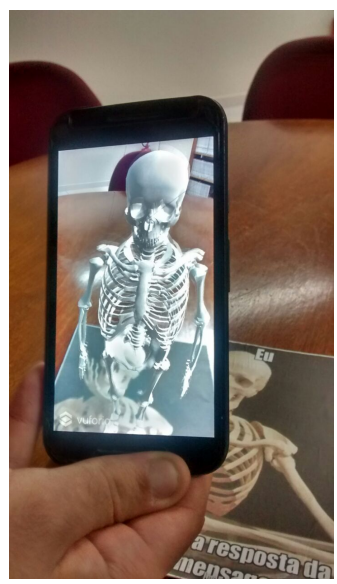

Figura 1. Visualização de um modelo em RA.

Portanto a tecnologia de RA consiste em possibilitar uma visualização tridimensional a partir de uma imagem com o auxílio de um dispositivo digital, como um smartphone ou tablet. A sobreposição de elementos virtuais pode ser realizada por meio de diferentes técnicas, dentre todas, a utilização de marcadores é a principal. Os marcadores são as imagens de referência do modelo 3D (três dimensões). 
VI Congresso Brasileiro de Informática na Educação (CBIE 2017)

Anais dos Workshops do VI Congresso Brasileiro de Informática na Educação (WCBIE 2017)

Os sistemas de RA são classificados em quatro estruturas: (i) visão ótica direta (óculos ou capacete), (ii) visão ótica por projeção (em que superfícies do mundo físico são usadas para a projeção de objetos virtuais), (iii) visão direta por vídeo (faz uso de câmera acoplada a capacete) e (iv) visão pautada em monitor (composto por uma webcam que realiza a captura da cena do mundo físico para misturá-la aos objetos virtuais e exibir o resultado em um monitor) [Azuma 2001].

\section{Desenvolvimento do RALTE}

O desenvolvimento do RALTE ocorreu durante três semanas, dividido em duas etapas. $\mathrm{Na}$ primeira etapa, foram realizadas pesquisas e estudos com propósito de conhecer sobre a temática de RA e as ferramentas para sua produção. Na segunda etapa, foi iniciado o desenvolvimento do aplicativo, voltado para práticas pedagógicas no LTE. No processo de desenvolvimento da aplicação foi utilizada uma versão gratuita do software Unity3D, um motor de jogo (game engine) 3D que facilita o desenvolvimento de jogos digitais, junto ao framework Vuforia ${ }^{1}$, um conjunto de códigos de programação comuns reutilizáveis para o desenvolvimento de aplicativos que envolvem RA. Com esses dois elementos foi possível, de maneira rápida e prática, desenvolver essa aplicação, sem a necessidade de conhecimento em programação, o que traz autonomia para qualquer um que tenha interesse em desenvolver aplicativos simples de RA.

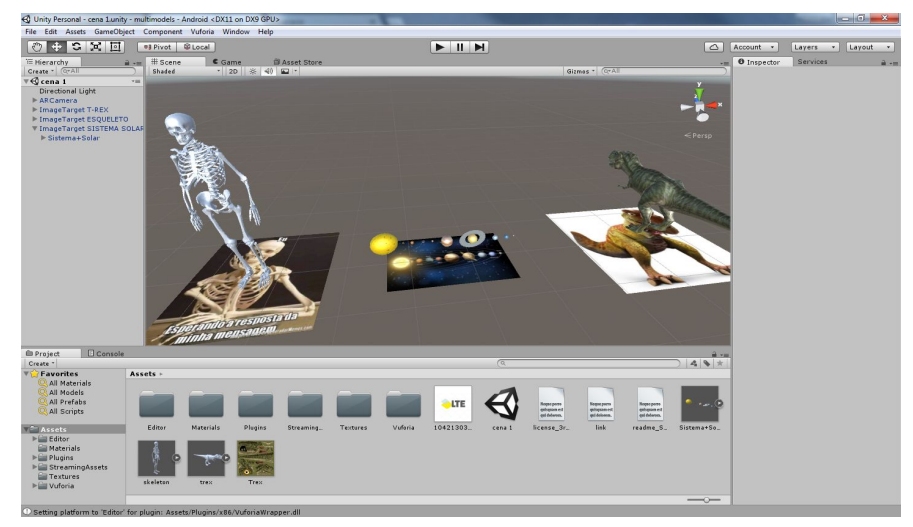

Figura 2 - Unity com o framework Vuforia

A figura 2 mostra a utilização da ferramenta Unity $3 D$ com os modelos 3D do aplicativo. Esse processo de desenvolvimento é rápido, porém pode variar de acordo com a quantidade de objetos 3D que o desenvolvedor quer exibir. Os objetos 3D utilizados (a caveira, o dinossauro e o sistema solar) foram retirados de bibliotecas de modelos, como por exemplo a biblioteca 3D Warehouse ${ }^{2}$ ou Free $3 d^{3}$, que permitem o download gratuito.

\footnotetext{
${ }^{1}$ Disponível em: <https://developer.vuforia.com/>. Acesso em: 30 Jun. 2017.

${ }^{2}$ Disponível em: <https://3dwarehouse.sketchup.com/>. Acesso em: 30 Jun. 2017.

${ }^{3}$ Disponível em: <https://free3d.com/>. Acesso em: 30 Jun. 2017.
} 
VI Congresso Brasileiro de Informática na Educação (CBIE 2017)

Anais dos Workshops do VI Congresso Brasileiro de Informática na Educação (WCBIE 2017)
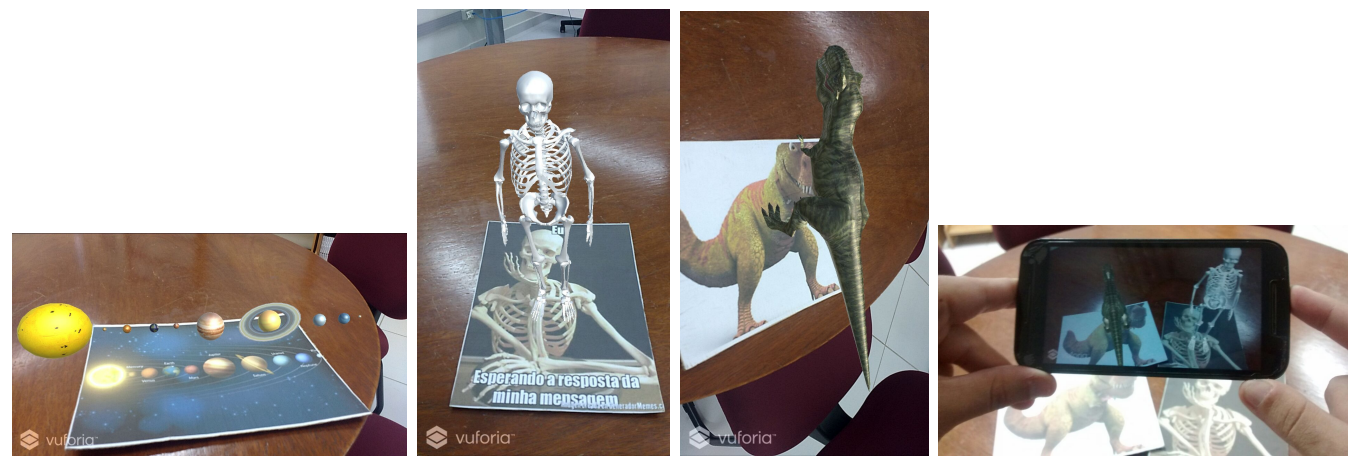

Figura 3 - Aplicações do RALTE.

É necessária a adição de marcadores aos respectivos modelos 3D, em seguida, é feita a compilação, que consiste na conversão do código-fonte em um arquivo executável do projeto no Unity3D, gerando um arquivo .apk ${ }^{4}$. Para utilizar o aplicativo é necessário apenas transferir o arquivo apk para um smartphone ou tablet e realizar a sua instalação. Por fim, basta abrir a aplicação e focar a câmera do dispositivo móvel nas imagens marcadoras.

\section{Considerações Finais}

Este trabalho mostrou que existe viabilidade em desenvolver e implantar aplicações de RA para tornar o aprendizado mais atrativo, significativo e contextualizado, e refletir sobre possibilidades de RA como uma interface que deve ser mais explorada no âmbito pedagógico.

É notável que RA é uma tecnologia crescente, que permite combinar realidade virtual e física, ocasionando uma imersão natural e motivadora ao usuário, fazendo com que aumente sua percepção sobre o tema trabalhado e gerando uma nova forma de aprendizado. Além disso, essa tecnologia permite novas formas de representação da realidade, diferentes níveis de interatividade e 'imersão perceptiva'.

Como fruto desse trabalho incipiente, temos a perspectiva de ampliar a produção de objetos educacionais em RA, disponibilizando esse recurso tecnológico para todos os níveis e áreas do conhecimento, sobretudo, na Educação Básica. Por isso, estão sendo realizados estudos e pesquisas para o desenvolvimento e uso de novas aplicações, tanto para professores como para alunos, no sentido de promover a inovação em tecnologia educacional.

\section{Referências}

Martins,V. F. \& Guimarães, M.P. (2012). "Desafios para o uso de Realidade Virtual e Aumentada de maneira efetiva no ensino". In: DEsafIE! - I Workshop de Desafios da Computação Aplicada à Educação - XXXII Congresso da Sociedade Brasileira de Computação, 2012, Curitiba.

\footnotetext{
${ }^{4}$ É um tipo de extensão de arquivo de um pacote de instalação de um aplicativo para sistema operacional Android, instalado no aparelho sem vínculo direto com a loja de aplicativos oficial, a PlayStore.
} 
VI Congresso Brasileiro de Informática na Educação (CBIE 2017)

Anais dos Workshops do VI Congresso Brasileiro de Informática na Educação (WCBIE 2017)

Yuen, Steve Chi-Yin, Gallayanee Yaoyuneyong, and Erik Johnson. (2013). "Augmented reality and education: Applications and potentials". Reshaping Learning. Springer Berlin Heidelberg, 385-414.

Silva, R., Jauvane C. Oliveira, and Gilson A. Giraldi. (2003). "Introduction to augmented reality". National Laboratory for Scientific Computation, Av. Getulio Vargas.

Azuma, R. T. (1997). “A Survey of Augmented Reality”, In Presence: Teleoperators and Virtual Environment, v. 6, n. 4, p. 355-385.

Azuma, R. et al. (2001). "Recents Advances in Augmented Reality". In IEEE Computer Graphics and Applications, vol. 21, p. 34-37. 